\title{
EFECTO DE LOS MÉTODOS DE REPRESENTACIÓN DE MOVIMIENTO SOBRE VARIABLES FUNCIONALES PROPIAS DEL BALONCESTO: UN ENSAYO CLÍNICO ALEATORIZADO PILOTO
}

\author{
María del Mar Ivars-Crespo, MSc ${ }^{1}$, Luis Suso-Martí, PhD 2,3,*, Aida Herranz-Gómez, MSc ${ }^{3}$, Álvaro Santamaría-Alveza, \\ PT 2, Ferran Cuenca-Martínez, $\mathrm{PhD}^{3}$ \\ 1. Hospital Universitario y Politécnico La Fe, Valencia, España. \\ 2. Departamento de Fisioterapia y Enfermería, Universidad CEU Cardenal Herrera, CEU Universities, Valencia, España. \\ 3. Motion in Brains Research Group, Institute of Neuroscience and Sciences of the Movement (INCIMOV). Centro Superior de Estudios Universitarios La \\ Salle, Universidad Autónoma de Madrid. 280323 Madrid (España).
}

\section{Correspondencia:}

Luis Suso-Martí, $\mathrm{PhD}$ Department of Physiotherapy, Universidad CEU Cardenal Herrera, CEU Universities,

Valencia, Spain. Teléfono: 961369000 E-mail: luis.suso@gmail.com

\section{Conflicto de Intereses:}

Los autores declaran no tener ningún conflicto de intereses

\section{Financiación:}

Este estudio no ha recibido ninguna subvención específica de organismos de financiación del sector público, comercial o sin ánimo de lucro.

DOI:

https://doi.org/10.37382/jomts.v3i1.468

\section{Recepción del Manuscrito:}

12-Junio-2021

Aceptación del Manuscrito:

21-Junio-2021

Licensed under: CC BY-NC-SA 4.0

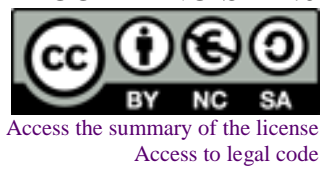

\section{RESUMEN}

Introducción: Se ha sugerido que tanto la imaginería motora (IM) como la observación de acciones (OA) son métodos efectivos en el aprendizaje de una acción motora determinada y para producir un aumento del rendimiento de ciertas habilidades motoras. Esto se debe a que comparten una base neurofisiológica común en el sistema de las neuronas espejo.

Objetivos: El objetivo del estudio fue determinar si la IM y la OA, combinadas con un programa de actividad física, son más efectivas en la mejora de las variables clave en el baloncesto como el salto, la precisión y la velocidad.

Métodos: Se realizó un ensayo clínico aleatorizado. Un total de 16 participantes asintomáticos fueron distribuidos en tres grupos de intervención diferentes: grupo placebo $(n=5)$, grupo de IM $(n=6)$ y grupo de OA $(n=5)$.

Resultados: El grupo de OA presentó un aumento en la velocidad ( $p<0,001$; $d=4,53 ; \quad(p<0,001, d=5.53)$, precisión $(p<0,001, d=3,47)$ y salto horizontal $(p<0,001, d=0,77)$. Los cambios en el grupo de IM destacaron mayoritariamente en el salto horizontal $(p<0,001, d=0,51)$. No se encontraron diferencias estadísticamente significativas entre los grupos de forma posterior a la intervención.

Conclusiones: La IM y la OA, junto con un programa específico de entrenamiento de baloncesto, resultaron ser intervenciones efectivas en la mejora de las diferentes habilidades estudiadas en la fase temprana del aprendizaje motor.

Palabras clave: Imaginería Motora; Observación de Acciones; Baloncesto. 


\section{INTRODUCCIÓN}

La imaginería motora (IM) es un proceso dinámico en el cual un sujeto simula una acción mentalmente sin realizar ningún movimiento $\mathrm{y}$, donde la representación de la acción específica es reproducida de manera interna en la memoria. Además, se puede dividir en IM cinestésica o IM visual (Decety, 1996). La observación de acciones (OA) se define como el proceso a través del cual una persona se expone a observar de manera visual un determinado gesto motor, sin realizar ningún movimiento, el cual evoca de forma interna una simulación del mismo (Gallese et al., 1996; Ge et al., 2018).

Desde el punto de vista neurofisiológico, ambas técnicas presentan efectos similares sobre el sistema nervioso. Se produce una activación de las neuronas espejo, situadas en las áreas parietal inferior, corteza premotora y motora primaria, que son clave en la ejecución y planificación del movimiento (Hardwick et al., 2018). Al imaginar u observar una acción motora se genera una activación cerebral similar a la que se produce con la ejecución de un movimiento (Jeannerod, 1994; Kilteni et al., 2018). Esta actividad neurofisiológica equivalente justifica el uso de dichas herramientas desde un punto de vista clínico, y estudios previos han mostrado su papel en la mejora de variables físicas o el aprendizaje motor (Mattar and Gribble, 2005; Herranz-Gómez et al., 2020).

Los métodos de representación de movimiento pueden ser muy útiles en las poblaciones de deportistas que han sufrido una lesión que les impida realizar determinados gestos deportivos. Es posible que la IM y la OA puedan mantener las habilidades motoras durante el periodo de la lesión, consiguiendo una posible disminución de los tiempos de recuperación y evitando la pérdida de habilidades deportivas clave como pueden ser la precisión, el salto o la velocidad. Además, la combinación de los métodos de representación del movimiento junto con la realización del movimiento de forma real podría mejorar tanto del rendimiento deportivo como del aprendizaje de un determinado gesto motor en deportistas noveles (Jeannerod, 1995; Munzert et al., 2008).
El objetivo principal de este estudio fue observar si la IM y la OA, en combinación con el ejercicio, pueden producir un efecto sobre variables funcionales críticas para el deporte de baloncesto en comparación con una intervención placebo. El objetivo secundario fue evaluar las diferencias entre ambas herramientas.

\section{MÉTODOS}

\section{Diseño del estudio}

Realizamos un ensayo clínico aleatorizado simple ciego, planificado y realizado de acuerdo con los requisitos de la declaración CONSORT (Schulz et al., 2010). El proyecto fue aprobado por el Comité de Ética para la Investigación Biomédica de la Universidad CEU-Cardenal Herrera (Número de aprobación: CEI20/004).

\section{Participantes}

Se reclutó una muestra de 16 voluntarios asintomáticos de la Comunidad Valenciana entre enero de 2019 y marzo de 2020. Los criterios de inclusión fueron los siguientes: (a) individuos asintomáticos y (b) hombres y mujeres 18 a 65 años. Los criterios de exclusión fueron: (a) conocimiento de las técnicas de representación del movimiento (IM y OA), (b) cualquier tipo de enfermedad neurológica o patología musculoesquelética del miembro superior y/o inferior y (e) deportistas que jueguen al baloncesto de manera profesional en el momento de su participación en el estudio. Todos los datos fueron registrados en el centro universitario CEU Cardenal Herrera de Alfara del Patriarca (Valencia).

Todos los participantes recibieron una explicación sobre los procedimientos del estudio, los cuales fueron planificados de acuerdo a las normas éticas de la Declaración de Helsinki. Se obtuvo el consentimiento informado por escrito de todos los participantes antes de su inclusión en el estudio.

\section{Aleatorización \\ La aleatorización se realizó empleando una tabla de secuencia aleatoria generada con un diseño de 3 bloques equilibrados (GraphPad Software, Inc., CA, USA). Un investigador independiente generó la lista}


de aleatorización y un miembro del equipo de investigación que no participó en la intervención ni en la evaluación de los participantes se encargó de la aleatorización. Los participantes incluidos se asignaron a uno de los tres grupos mediante la lista de secuencia aleatoria, garantizando así la ocultación de la asignación.

\section{Cegamiento}

Las evaluaciones e intervenciones fueron realizadas por investigadores diferentes. El evaluador estaba cegado a la asignación del participante en el momento de las mediciones y registro de los datos, por lo cual no supo en ningún momento la intervención que había recibido cada participante. Se pidió a los participantes que no hicieran ningún comentario al investigador que realizaba las mediciones.

\section{Intervenciones}

\section{Grupo placebo}

Los participantes del grupo placebo (GP) realizaron un programa de entrenamiento de baloncesto que incluyó diferentes ejercicios dirigidos a obtener una mejora en algunas habilidades del baloncesto, incorporando ejercicios de velocidad, con y sin bote de balón, salto vertical y precisión en tiros libres. Todas las sesiones fueron dirigidas por un fisioterapeuta para guiar en la correcta ejecución de los ejercicios.

El primer ejercicio, de velocidad, consistió en realizar un recorrido de un lado a otro de la pista, tomando como referencia el semicírculo de la línea de triples, realizando dicho recorrido en el menor tiempo posible. Realizaron 4 repeticiones, dos sin balón y dos combinando la acción de correr con la de botar la pelota. El segundo ejercicio, de precisión, consistió en realizar 10 tiros a canasta desde la posición de tiro libre. El tercer ejercicio, de salto, se basó en ejecutar tres saltos verticales consecutivos con el objetivo de alcanzar la máxima altura posible. Entre cada uno de los ejercicios el participante observó un video placebo, en el que se representaban escenas sin agentes humanos.

\section{Grupo de imaginería motora}

Los participantes del grupo de IM realizaron una primera fase de entrenamiento idéntica a la del GP, en la que realizaron los ejercicios de baloncesto de manera activa.

Además, posteriormente realizaron una segunda fase que consistió en un entrenamiento de IM. Se llevó a cabo una intervención basada en IM cinestésica en primera persona, en la que cada participante se imaginaba a sí mismo realizando los mismos ejercicios del protocolo de entrenamiento ejecutado anteriormente, sintiendo cada uno de los gestos motores realizados, involucrando así el sistema somatosensorial. Todas las actividades de IM fueron guiadas por el fisioterapeuta durante 1 minuto.

\section{Grupo de observación de acciones}

Los participantes del grupo de OA realizaron una primera fase idéntica a la del GP, es decir, realizando los ejercicios del entrenamiento de baloncesto de manera activa.

Posteriormente, realizaron una segunda fase de entrenamiento basado en la OA, empleando una perspectiva en tercera persona. Los participantes visualizaron un vídeo, en el que otra persona realizaba cada uno de los ejercicios del protocolo de entrenamiento ejecutados anteriormente.

\section{Procedimiento}

Después de dar su consentimiento para participar en el estudio y antes del comienzo de la intervención, los participantes recibieron un conjunto de cuestionarios, incluyendo información sociodemográfica, así como la evaluación del nivel de actividad física y capacidad de generar imágenes motoras mentales.

Una vez se completó dicha evaluación, se realizó la evaluación pre-intervención. Los participantes realizaron una única sesión de intervención según el grupo al que fueron asignados. Al finalizar la intervención, se realizó la evaluación postintervención. En las evaluaciones se cuantificó la capacidad de precisión en los tiros libres, de salto horizontal y velocidad con o sin bote de balón. 


\section{Variables}

Variables principales

Velocidad. El T-Test se empleó para evaluar la velocidad y la agilidad, puesto que involucra el cambio direccional. Se utiliza un circuito en forma de $\mathrm{T}$, delimitado por puntos de referencia del campo de baloncesto: (1) línea de medio campo, (2) punto central de la línea de tiros libres, (3) lado derecho de la línea de tiros libres, (4) lado izquierdo de la línea de tiros libres. Primero se realiza el recorrido corriendo y, después, incorporando la acción de botar el balón, incluyendo así su capacidad de coordinación. Se pidió a los participantes que realizaran el recorrido en el menor tiempo posible. Se evaluó el tiempo que cada participante tardó en realizar el recorrido.

Precisión. La precisión se evaluó mediante la prueba de 10 tiros libres. Cada participante efectuó 10 tiros libres de forma consecutiva desde la posición del centro de la línea que corresponde a este punto. Se cuantificó la cantidad de aciertos.

Salto horizontal. Se evaluó la capacidad de salto en el eje horizontal. Los participantes se situaron en una línea utilizada como referencia del punto inicial. A continuación, con los pies alineados realizaron un salto horizontal, con el objetivo de alcanzar la mayor distancia posible respecto al punto inicial. El evaluador, empleando una cinta métrica, evaluó la distancia entre el punto inicial (línea de referencia) y el punto final (posición del talón del pie que hubiera quedado posterior respecto al contralateral). Se realizaron 3 saltos consecutivos con un descanso entre ellos para evitar el efecto de la fatiga, registrando la repetición en la que la distancia fuera mayor.

\section{Variables basales}

Nivel de actividad física. El nivel de actividad física se evaluó empleando el cuestionario The International Physical Activity Questionnaire (IPAQ), que ha mostrado validez aceptable en aquellos estudios que miden la actividad física total. Se subdivide en actividades físicas intensas o moderadas, caminar e inactividad, y clasifica a los participantes en tres grupos en función de su nivel de actividad física (alto, moderado y bajo/sedentario) (Roman-Viñas et al., 2010).

Capacidad de generar imágenes motoras mentales. La capacidad de imaginación se cuantificó mediante el cuestionario The Movement Imagery Questionnaire-Revised (MIQ-R). Consta de 4 movimientos repetidos en dos dominios (visual y cinestésico). Los participantes evaluaron la dificultad o facilidad de visualizar/sentir las imágenes motoras en una escala de 7 puntos, donde el 1 corresponde con la máxima dificultad y el 7 con la máxima facilidad para ver/sentir las imágenes mentales motoras (Campos A. y González MA., 2010).

\section{Análisis estadístico}

El análisis estadístico se realizó empleando Paquete Estadístico para las Ciencias Sociales (SPSS 22, SPSS Inc., Chicago, IL, EE.UU.) y se evaluó la normalidad de las variables utilizando la prueba de Shapiro-Wilk. Para resumir los datos de las variables continuas, se utilizaron estadísticos descriptivos y se presentan los resultados como media \pm desviación estándar, 95\% del intervalo de confianza. Las variables categóricas se presentan como frecuencias absolutas (número) y relativas (porcentaje). Para comparar las variables categóricas se utilizó una prueba de chi cuadrado con análisis residual. Para comparar las variables de resultado continuo fue empleado un análisis de varianza de medidas repetidas de dos vías (ANOVA). Los factores analizados fueron los grupos (GP, IM y OA) y los tiempos (pre y post intervención). También fue analizada la interacción tiempo*grupo. Se calculó la eta-cuadrado parcial $\left(\eta \mathrm{p}^{2}\right)$ como medida del tamaño del efecto (fuerza de asociación) para cada efecto principal y la interacción en los ANOVAs $(0,01-$ 0,059 representa un efecto pequeño; 0,06-0,139 representa un efecto medio; y $>0,14$ representa un efecto grande). Se realizó un análisis post hoc con corrección de Bonferroni en el caso de hallazgos significativos de ANOVA para comparaciones múltiples entre variables. El tamaño del efecto $(d)$ se calculó según el método de Cohen, en el que la magnitud del efecto se clasificó como pequeña $(0,20$ - 
$0,49)$, mediana $(0,50-0,79)$ o grande $(0,8)$. El nivel de $\alpha$ se fijó en 0,05 para todas las pruebas (Cohen, 2013).

\section{RESULTADOS}

Un total de 16 participantes fueron incluidos y distribuidos al azar en 3 grupos de intervención $(\mathrm{GP}=5 ; \mathrm{IM}=6 ; \mathrm{OA}=5)$. No hubo efectos adversos en ninguno de los participantes. No hubo diferencias estadísticamente significativas en las variables demográficas ni en las variables basales de estudio antes de la intervención entre grupos (Tabla 1). pre-intervención y post-intervención con un tamaño de efecto grande en el grupo de OA $(p<0,001$, $d=5.53) \mathrm{y}$, mediano en el GP $(p=0,018, d=0,5)$ y en grupo de IM $(p=0,004, d=0,67)$. La diferencia más grande se aprecia en el grupo de observación (OA) respecto al resto. Sin embargo, esta diferencia no resulta ser significativa al realizar una comparación entre los grupos (Figura 2).

\section{Precisión}

La prueba ANOVA mostró cambios estadísticamente significativos en los tiros libres tanto en la interacción tiempo*grupo $(\mathrm{F}=8,27$;

Tabla 1. Características sociodemográficas y de las variables basales.

\begin{tabular}{lllc} 
Medidas & \multicolumn{1}{c}{ GP (n=5) } & \multicolumn{1}{c}{ OA (n=5) } & IM (n=6) \\
Edad (años) & $24,20 \pm 4,76$ & $30,00 \pm 10,93$ & $22,50 \pm 1,52$ \\
Género & & & 0,199 \\
$\quad$ Masculino & $4(80)$ & $4(80)$ & $2(33,33)$ \\
$\quad 1(20)$ & $1(20)$ & $4(66,66)$ & $1,74 \pm 0,085$ \\
$\quad$ Femenino & $1,77 \pm 0,072$ & $1,79 \pm 0,065$ & $64,50 \pm 10,75$ \\
Altura & $71,60 \pm 10,07$ & $69,60 \pm 6,58$ & $3147,75 \pm 907,32$ \\
Peso & $2907,80 \pm 2327,86$ & $1804,00 \pm 643,48$ & 0,416 \\
MIQ-R & $52,40 \pm 2,07$ & $52,00 \pm 2,83$ & 0,311
\end{tabular}

IPAQ: Cuestionario Internacional de Actividad Física; MIQ-R: Cuestionario de imagen de movimiento revisado.

\section{Velocidad}

La prueba ANOVA mostró cambios estadísticamente significativos en el T-test tanto en la interacción tiempo*grupo $\quad(\mathrm{F}=8,26 ; \quad p=0,005$; $\left.\eta \rho^{2}=0,56\right)$ como en el tiempo $(\mathrm{F}=40,12 ; p<0,001$; $\left.\eta \rho^{2}=0,75\right)$. La prueba post-hoc únicamente reveló diferencias significativas entre la medición preintervención y la medición post-intervención en el grupo de OA con un tamaño de efecto grande $(p<0,001 ; d=4,53)$, pero no en el grupo IM $(p=0,057)$ ni en el GP $(p=0,075)$. Sin embargo, no hubo diferencias significativas entre los grupos después de la intervención (Figura 1).

En relación con el T-Test con bote de balón, la prueba ANOVA mostró cambios estadísticamente significativos en la interacción tiempo*grupo ( $\left.\mathrm{F}=4,56, p=0,032, \eta \rho^{2}=0,41\right)$ como en el tiempo $\left(\mathrm{F}=55,79, p<0,001, \eta \rho^{2}=0,81\right)$. La prueba post-hoc reveló diferencias significativas entre la medición $\left.p=0,005 ; \eta \rho^{2}=0,56\right)$ como en tiempo $(\mathrm{F}=14,226$; $\left.p=0,002 ; \eta \rho^{2}=0,523\right)$. La prueba post hoc únicamente

Figura 1. Diferencias entre las evaluaciones pre y postintervención en la velocidad (T-Test).

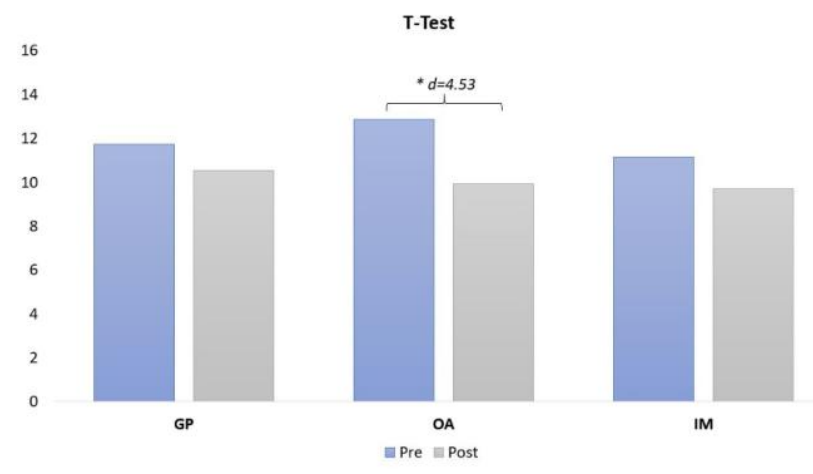

${ }^{*} \mathrm{p}<0,05 ; \mathrm{d}$ : d de Cohen; GP: Grupo placebo; IM: Imaginería motora; OA: Observación de acciones

reveló diferencias significativas entre la medición pre-intervención y la medición post-intervención, en 
el grupo OA con un tamaño de efecto grande $(p<0,001, d=3,47)$, pero no en el GP $(p=0,725)$ e IM $(p=0,523)$. Sin embargo, no se encontraron diferencias entre los grupos de forma posterior a la intervención (Figura 3).

Figura 2. Diferencias entre las evaluaciones pre y postintervención en la velocidad con bote de balón (T-Test Bote)

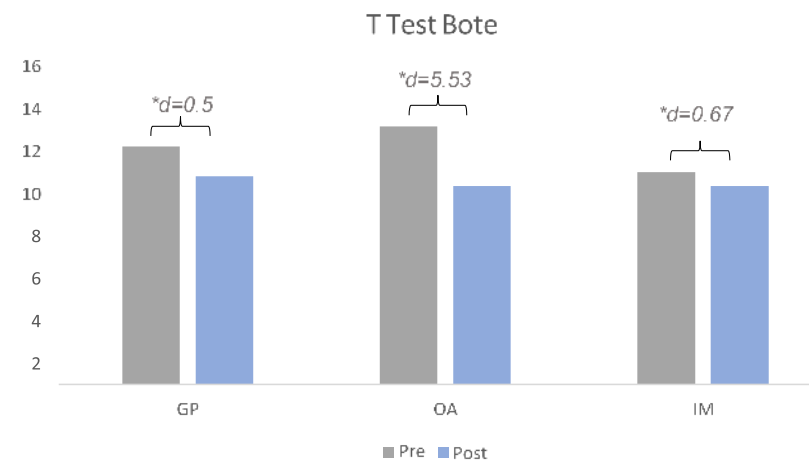

*p<0,05; d: d de Cohen; GP: Grupo placebo; IM: Imaginería motora; OA: Observación de acciones

\section{Salto horizontal}

La prueba ANOVA mostró cambios estadísticamente significativos en la variable salto únicamente en la interacción tiempo $(\mathrm{F}=50,32 ; p<$ 0,$\left.001 ; \eta \rho^{2}=0,79\right)$, pero no en la interacción tiempo*grupo $\left(\mathrm{F}=3,65 ; \quad p=0,055 ; \eta \rho^{2}=0,36\right) . \quad \mathrm{La}$ prueba post hoc reveló diferencias significativas entre la medición pre-intervención y post-intervención, con un tamaño de efecto mediano en los grupos OA $(p<0,001, d=0,77)$ e IM $(p<0,001, d=0,51)$, pero no en el GP $(p=0,094)$. Sin embargo, no hubo diferencias significativas entre grupos en la medición post-intervención (Figura 4).

\section{DISCUSIÓN}

El objetivo principal del presente estudio fue evaluar si la IM y la OA, en combinación con el ejercicio real, influyen sobre variables funcionales críticas para el deporte de baloncesto, en comparación con una intervención placebo. Las pruebas T-Test y Tiros libres, que evaluaron la velocidad y precisión respectivamente, mostraron diferencias estadísticamente significativas entre la medición pre-intervención y post-intervención únicamente en el grupo OA. La prueba T-Test Bote mostró diferencias significativas en los tres grupos de intervención. En la prueba de salto horizontal, se obtuvieron resultados estadísticamente significativos en el grupo OA e IM.

Estudios previos en este campo sugieren que el entrenamiento de OA e IM es efectivo en el aprendizaje o reaprendizaje de un determinada función motora y en la mejora de ciertas variables funcionales en determinados deportes (Robin et al., 2007; Di Rienzo et al., 2019). La equivalencia funcional a nivel neurofisiológico sugiere que los métodos de representación de movimiento y el ejercicio real producen efectos similares sobre el sistema nervioso, produciendo una activación de las neuronas espejo y, por consiguiente, recreando una estimulación cerebral similar a la que se produce con la ejecución de un movimiento real (Hétu et al., 2013).

Por otro lado, el grupo de AO mostró resultados más relevantes al comparar la evaluación pre y post intervención respecto a los otros grupos. Específicamente estos resultados se encontraron en la prueba T-Test, sin y con bote, así como en la prueba de tiros libres. Estos hallazgos son consistentes con estudios previos en este campo, dónde se han encontrado resultados más positivos en el tiempo en el grupo de OA (Gatti et al., 2013; Gonzalez-Rosa et al., 2015). El entrenamiento específico de baloncesto en combinación con la OA mostró mejores resultados al comparar las evaluaciones pre y post-intevención

Figura 3. Diferencias entre las evaluaciones pre y postintervención en la precisión.

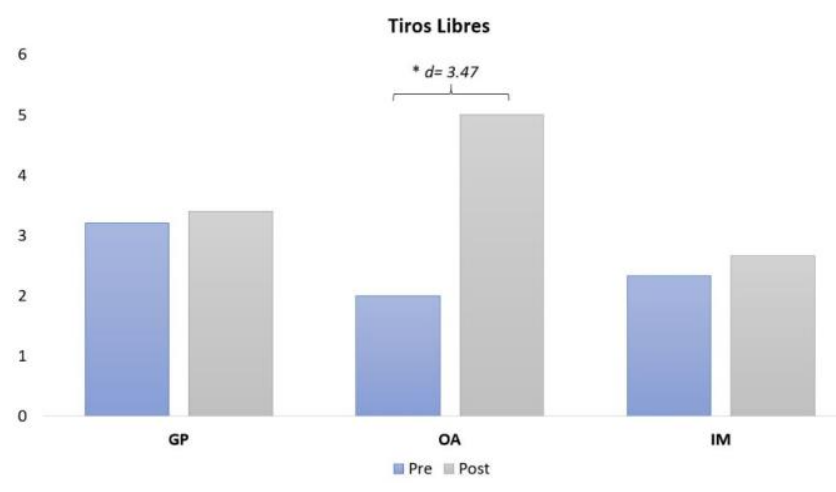

*p<0,05; d: d de Cohen; GP: Grupo placebo; IM: Imaginería motora; OA: Observación de acciones 
respecto a los grupos de IM y GP, en cuanto a velocidad con y sin bote de balón y precisión, sin embargo, la $\mathrm{OA}$ no fue superior a las otras intervenciones.

El éxito de la OA podría deberse a que la mayoría de los participantes no eran jugadores profesionales de baloncesto y el entrenamiento mediante OA ofrece un estímulo visual externo, en el que se muestra un contexto y ejecución de la acción adecuados. La visualización de una ejecución correcta en el vídeo podría facilitar posteriormente la recreación de ese ejercicio. Sin embargo, la IM precisa la creación de la imagen motora mental por parte del participante y, por tanto, depende de su capacidad para crear dichas imágenes mentales (Robin et al., 2007; ParisAlemany et al., 2019).

Figura 4. Diferencias entre las evaluaciones pre y postintervención en el salto horizontal.

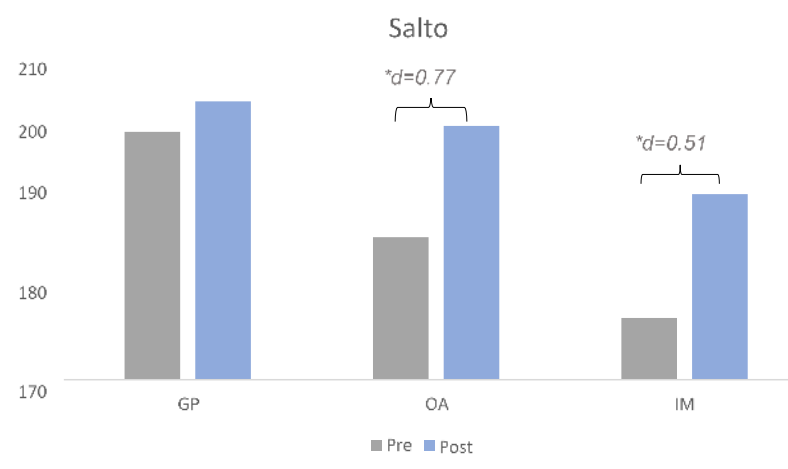

${ }^{*} \mathrm{p}<0,05$; d: d de Cohen; GP: Grupo placebo; IM: Imaginería motora; OA: Observación de acciones

\section{Limitaciones}

Existen varias limitaciones que es necesario tener en cuenta en la interpretación de los resultados. En primer lugar, es necesario destacar que es un estudio piloto con un pequeño tamaño muestral. En segundo lugar, únicamente muestra el resultado de una intervención a corto plazo mediante una única sesión de entrenamiento, por tanto se puede evaluar su efecto sobre el aprendizaje motor temprano, pero sería necesario evaluar si estos cambios se mantienen en el tiempo. En tercer lugar, se trata de una muestra asintomática y, por tanto, sería necesario evaluar el efecto de las técnicas de representación del movimiento y su impacto en población clínica.

\section{CONCLUSIÓN}

Tanto la IM como la OA, en combinación con el entrenamiento físico, pueden ser estrategias de entrenamiento útiles en la mejora de diferentes habilidades motoras y del proceso aprendizaje motor de acciones propias del baloncesto. Aunque no hubo diferencias significativas entre los grupos, parece que la OA presentó mayores cambios en el tiempo, al comparar las intervenciones pre y post-intervención. Es necesario que futuros estudios evalúen el impacto de estas herramientas en la rehabilitación de gestos deportivos o en contextos clínicos.

\section{FRASES DESTACADAS}

$>$ La imaginería motora y la observación de acciones, en combinación con el entrenamiento físico, mejoraron habilidades motoras del baloncesto.

$>$ Las técnicas de representación del movimiento presentaron resultados favorables a corto plazo en cuanto a velocidad, precisión y salto.

$>$ La observación de acciones presentó mayores cambios que la imaginería motora y el grupo placebo entre las evaluaciones pre y postintervención, pero no hubo diferencias significativas entre los grupos.

\section{REFERENCIAS}

Campos A. y González MA. Spanish version of the revised movement image questionnaire (miq-r): psychometric properties and validation. Rev Psicol del Deport. 2010;19(2):263-73.

Cohen J. Statistical Power Analysis for the Behavioral Sciences. Statistical Power Analysis for the Behavioral Sciences. 2013. DOI: http://dx.doi.org/10.4324/9780203771587.

Decety J. The neurophysiological basis of motor imagery. Behav Brain Res. 1996;77(1-2):45-52.

Gallese V, Fadiga L, Fogassi L, Rizzolatti G. Action recognition in the premotor cortex. Brain. 1996;119(2):593-609 DOI: http://dx.doi.org/10.1093/brain/119.2.593.

Gatti R, Tettamanti A, Gough PM, Riboldi E, Marinoni L, Buccino G. Action observation versus motor imagery in learning a complex motor task: A short review of literature 
and a kinematics study. Neurosci Lett. 2013;540:37-42 DOI: http://dx.doi.org/10.1016/j.neulet.2012.11.039.

Ge S, Liu H, Lin P, Gao J, Xiao C, Li Z. Neural Basis of Action Observation and Understanding From First- and ThirdPerson Perspectives: An fMRI Study. Front Behav Neurosci. Frontiers; 2018;12:283 DOI: http://dx.doi.org/10.3389/fnbeh.2018.00283.

Gonzalez-Rosa JJ, Natali F, Tettamanti A, Cursi M, Velikova S, Comi G, Gatti R, Leocani L. Action observation and motor imagery in performance of complex movements: Evidence from EEG and kinematics analysis. Behav Brain Res. Elsevier B.V.; 2015;281:290-300 DOI: http://dx.doi.org/10.1016/j.bbr.2014.12.016.

Hardwick RM, Caspers S, Eickhoff SB, Swinnen SP. Neural correlates of action: Comparing meta-analyses of imagery, observation, and execution. Neurosci Biobehav Rev. 2018;94:31-44

DOI: http://dx.doi.org/10.1016/j.neubiorev.2018.08.003.

Herranz-Gómez A, Gaudiosi C, Angulo-Díaz-Parreño S, SusoMartí L, La Touche R, Cuenca-Martínez F. Effectiveness of motor imagery and action observation on functional variables: An umbrella and mapping review with metameta-analysis. Neurosci Biobehav Rev. Elsevier Ltd; 2020;118:828-45

DOI: http://dx.doi.org/10.1016/j.neubiorev.2020.09.009.

Hétu S, Grégoire M, Saimpont A, Coll M-P, Eugène F, Michon P-E, Jackson PL. The neural network of motor imagery: An ALE meta-analysis. Neurosci Biobehav Rev. 2013;37(5):930-49 DOI: http://dx.doi.org/10.1016/j.neubiorev.2013.03.017.

Jeannerod. The representing brain: Neural correlates of motor intention and imagery. Behav Brain Sci. Cambridge University Press; 1994;17(2):187-202 DOI: http://dx.doi.org/10.1017/S0140525X00034026.

Jeannerod M. Mental imagery in the motor context. Neuropsychologia. 1995;33(11):1419-32 DOI: http://dx.doi.org/10.1016/0028-3932(95)00073-C.

Kilteni K, Andersson BJ, Houborg C, Ehrsson HH. Motor imagery involves predicting the sensory consequences of the imagined movement. Nat Commun. Nature Publishing Group; 2018;9(1):1-9 DOI: http://dx.doi.org/10.1038/s41467-018-03989-0.

Mattar AAG, Gribble PL. Motor Learning by Observing. Neuron. 2005;46(1):153-60 DOI: http://dx.doi.org/10.1016/j.neuron.2005.02.009.

Munzert J, Zentgraf K, Stark R, Vaitl D. Neural activation in cognitive motor processes: Comparing motor imagery and observation of gymnastic movements. Exp Brain Res. Exp Brain Res; 2008;188(3):437-44 DOI: http://dx.doi.org/10.1007/s00221-008-1376-y.

Paris-Alemany A, La Touche R, Gadea-Mateos L, CuencaMartínez F, Suso-Martí L. Familiarity and complexity of a movement influences motor imagery in dancers: A crosssectional study. Scand J Med Sci Sports. 2019;29(6):897906 DOI: http://dx.doi.org/10.1111/sms.13399.

Di Rienzo F, Joassy P, Kanthack T, MacIntyre TE, Debarnot U,
Blache Y, Hautier C, Collet C, Guillot A. Effects of Action Observation and Action Observation Combined with Motor Imagery on Maximal Isometric Strength. Neuroscience. Elsevier Ltd; 2019;418:82-95 DOI: http://dx.doi.org/10.1016/j.neuroscience.2019.08.025.

Robin N, Dominique L, Toussaint L, Blandin Y, Guillot A, Her $M$ Le. Effects of motor imagery training on service return accuracy in tennis: The role of imagery ability. Int J Sport Exerc Psychol. Informa UK Limited; 2007;5(2):175-86 DOI: http://dx.doi.org/10.1080/1612197x.2007.9671818.

Roman-Viñas B, Serra-Majem L, Hagströmer M, Ribas-Barba L, Sjöström M, Segura-Cardona R. International Physical Activity Questionnaire: Reliability and validity in a Spanish population. Eur J Sport Sci. 2010;10(5):297-304 DOI: http://dx.doi.org/10.1080/17461390903426667.

Schulz KF, Altman DG, Moher D. CONSORT 2010 Statement: Updated guidelines for reporting parallel group randomised trials. BMC Med. BioMed Central; 2010;8(1):18 DOI: http://dx.doi.org/10.1186/1741-7015-8-18. 